Chester Luiz Galvão Cesar 1

\title{
Inquérito domiciliar como instrumento de avaliação de serviços de saúde: um estudo de caso na região sudoeste da área metropolitana de São Paulo, 1989-1990
}

\author{
Household surveys as an evaluation tool for health \\ services: a case study from the Southeast region \\ of the Greater São Paulo Metropolitan Area,
} 1989-1990
1 Departamento de Epidemiologia, Faculdade de Saúde Pública, Universidade de São Paulo, Av. Dr. Arnaldo 715, São Paulo, SP, 01246-904, Brasi

2 Departamento de Saúde Materno Infantil,

Faculdade de Saúde Pública, Universidade de São Paulo, Av. Dr. Armando 715,

São Paulo, SP,

01246-904, Brasi
Abstract The increasing importance of health eval uation has raised the need of incorporating epidemiological methodology to assess both technologi es and health services. Evaluation of health services performance requires population data to identify the unmet demand and patterns of health services utilization. Such data can be obtained through hou sehold surveys. A household survey in the Greater São Paulo Metropolitan Area identified the pattern of health services utilization by the local population. This pattern, which reflects current organization of health services, pointed to a low coverage level by local services, including public clinics, different profiles for users of private and public services, and a large demand for health services outsi de the area, mainly for childbirth. The information obtained through these surveys produces a "base line" for health planning and further evaluation and is central to the assessment of existing services. The advantages of household surveys in health services evaluation should be analyzed in relation to the potential use of such data and study costs, thereby desi gning the best cost-ben efit relationship for each specific situation.

Key words Epidemiology; Health Services Evalluation; Household Surveys; Health Services

Resumo A importância crescente da avaliação em saúde requer a incorporação do método epidemi ológico, tanto no processo de avaliação de tecnologias, como na avaliação do desempenho dos servi ços de saú de. Esta aval iação necessita de informações com base populaci onal, que identifiquem a demanda reprimi da e caracterizem o padrão de utilização de serviços, as quais podem ser obti das através de inquéritos domi ciliares. As i nformações geradas através destes inquéritos não só permitem uma "Iinha de base" para o planejamento de saúde e posteri or aval iação, como também são i mportantes na aval iação dos serviços. Inquérito domi ciliar real izado na Grande São Paulo permitiu identificar o padrão de uso dos serviços de saúde para a população residente na área. Este padrão reflete a organização dos serviços de saúde, mostrando a baixa cobertura dos servi ços l ocais, incl usive a dos serviços públi cos, os di ferentes perfis dos usuários dos serviços privados e públi cos e a grande procura de serviços fora da área, no Município de São Paulo, principalmente para o parto. As vantagens de incorporar inquéritos domi ciliares no processo de avaliação de servi ços devem ser anal isadas frente ao uso potencial dos resultados e aos custos destes estudos, devendo ser discutido, em cada si tuação específica, o del ineamento mais adequado para uma mel hor relação custo-benefício.

Palavras-chave Epidemiologia; Avaliação de Saúde; Inquéritos Domiciliares; Serviços de Saúde 
Inquérito domiciliar como instrumento de avaliação de serviços de saúde

A avaliação em saúde vem apresentando uma importância crescente na últimas décadas, possivelmente em decorrência da incorporação contínua, pelo trabalho médico, de novas tecnologias, e também pela ampliação e crescente complexidade dos serviços médicos, o que ocorreu juntamente com o processo de reformas sociais, a partir do final da década de 40, principalmente nos países europeus.

Esta necessidade de avaliação em saúde vem aumentando a necessidade de incorporação do método epidemiológico, tanto no processo de avaliação de tecnologias como no de análise de desempenho dos serviços de saúde.

A necessidade de avaliação das novas tecnologias disponíveis prende-se a dois aspectos de fundamental importância: o primeiro corresponde à necessidade de se optar por aquela que apresente a maior eficácia e os menores efeitos colaterais; o segundo diz respeito à necessidade de se estabelecer a relação custo-benefício, dado o crescente custo dessas tecnologias incorporadas, cuja eficácia, muitas vezes, não se conhece com precisão e objetividade. A metodologia para essa avaliação de eficácia foi amplamente difundida e incorporada pelo setor saúde, principalmente através dos ensaios clínicos. A divulgação e a disseminação do uso de novos procedimentos médicos ou novas drogas baseia-se, com maior freqüência, na avaliação dos resultados desses ensaios clínicos, que são possíveis de ser realizados com razoável grau de objetividade e precisão.

Na maioria dos países, a ampliação e a grande complexidade organizacional dos serviços de saúde também estão a exigir um processo objetivo de avaliação em função de seus custos e do aumento constante da demanda. Essa avaliação é possivelmente menos objetiva e precisa que a anterior.

Várias definições de avaliação têm sido apresentadas na tentativa de organizar e facilitar essa avaliação dos serviços de saúde.

Assim, segundo a Organização Mundial da Saúde (1989), a avaliação é definida como "um meio sistemático de se aprender empiricamente e de se utilizar as lições aprendidas para meIhoria das atividades realizadas e para o desenvolvimento de um planejamento mais satisfatório, mediante uma seleção rigorosa entre as distintas possibilidades de ação futura".

Já Pabon (1985) define como - "medir um fenômeno ou o desempenho de um processo, comparar o resultado obtido com os critérios es- tabelecidos, e fazer uma análise crítica conside rando-se a magnitude e a direção da diferença".

Para Calsing (1982), "a avaliação tem de ser aplicada nos vários momentos da administração com a finalidade de produzir informações capazes de confirmar ou modificar as próprias decisões dentro do planejamento, funcionando como dispositivos auto reguladores do sistema".

Em resumo, avaliar é medir as condições existentes, o processo de trabalho e os resultados obtidos, fazendo as comparações tanto com as condições e as tecnologias previstas, quanto com os resultados e metas a serem alcançados, para a emissão de um juízo de valor.

A avaliação é constituída de etapas que podem ser agrupadas nas ações de - medir, comparar e emitir juízo de valor.

Para se iniciar a avaliação, torna-se imprescindível identificar a amplitude e a legitimidade das decisões possíveis de serem tomadas, sem as quais pode ocorrer a perda da finalidade última da avaliação, qual seja a de emitir um juízo que permita a tomada de decisão. Portanto, a avaliação no campo da saúde exige uma prévia formulação de hipóteses, que deverão estar baseadas no conhecimento existente e disponível sobre o objeto a ser avaliado, e que também deverão direcionar o conteúdo e a escolha dos instrumentos a serem utilizados na aval iação. Em geral, a formulação de hi póteses deve levar em consideração o contexto mediato e imediato, uma vez que estes delimitam a amplitude das decisões possíveis de serem tomadas.

Outro aspecto a ser salientado na avaliação é o grau de subjetividade a ela inerente, na medida em que o juízo de valor depende da visão de mundo de quem a executa. A aceitação de certo grau de subjetividade, inerente ao processo de avaliação enfocado na dinâmica dos fenômenos sociais, que caracteriza o setor saúde, resulta na necessidade de se aceitar que não há uma abordagem correta ou uma metodologia ideal para a avaliação, e sim uma composição de métodos e instrumentos organizados de forma a se atingir o objetivo de emitir um juízo de valor em relação ao objeto ou sujeito social avaliado.

Portanto, a avaliação constitui uma etapa essencial do processo de planejamento e de administração do setor saúde, uma vez que possibilita a tomada de decisões da maneira mais racional possível em face dos problemas enfrentados pelos serviços de saúde.

A avaliação necessita identificar com clareza o que vai avaliar, que características observar e medir, como pretende observar e medir esse sujeito ou objeto e o que fará com o juízo de valor emitido nesse processo. Assim, a ava- 
liação depende de quem a realizará e para quem se destina, isto é, com a avaliação realizada, que decisões serão tomadas e por quem.

A avaliação com enfoque sistêmico e caráter administrativo tem sido a mais amplamente utilizada nos serviços de saúde, particularmente aquela baseada na abordagem preconizada por Donabedian (1980). Esse autor propõe três componentes para a avaliação - Estrutura, Processo e Resultado.

O componente Estrutura corresponde à avaliação dos recursos existentes para a execução dos serviços. Esses recursos são: recursos físicos, compreendendo planta física, equipamentos e materiais de consumo; recursos humanos, compreendendo número e qualificação dos mesmos; fontes e recursos financeiros para manutenção da infra-estrutura e da tecnologia disponível. Este último compreende o conhecimento disponível e organizado como processo de trabalho, que viabilize a otimização da utilização dos outros recursos disponíveis na Estrutura. Nesse sentido, Frenk \& Mohr (1985) reforçam que "a tecnologia disponível permite modificar parcialmente a evolução natural das doenças, definindo a qualidade da atenção em termos de resultados obtidos".

O componente Processo corresponde à avaliação da execução das atividades e da dinâmica apresentada na inter-relação entre as aquelas. A análise das relações entre as atividades deverá possibilitar a avaliação da utilização da tecnologia prevista na Estrutura, para ser aplicada na execução. Na medida em que o processo de trabalho do setor saúde implica necessariamente a efetiva participação do usuário para a obtenção de um produto, fica claro que não é possível a avaliação do processo sem o envolvimento deste usuário, a quem se destina cada atividade do processo de forma imediata durante a execução. Para a avaliação do uso da tecnologia no Processo, será necessária a prédefinição de critérios ou de parâmetros, que poderão ser implícitos ou explícitos aos provedores dos serviços. Desta forma, pode-se analisar quão adequadas e apropriadas ao cliente foram as ações executadas.

O componente Resultado refere-se aos efeitos ou aos produtos das ações realizadas pelos serviços de saúde, que modificaram a situação de saúde dos usuários ou da comunidade. Levando-se em conta que situações de saúde se modificam devido a outros fatores sociais, é necessário o conhecimento prévio da situação para que se possa atribuir a modificação observada ao processo de intervenção objeto da avaliação.

Segundo Constanzo \& Vertinsky (1975), que trabalharam na abordagem de Donabedian, o
Resultado pode ser separado em aspectos "fisiopatológicos" - quando referentes a efeitos concretos na história natural das doenças - e em "psicossociais" - quando se referem aos efeitos resultantes do relacionamento social ocorrido entre os provedores e os usuários, resultados esses que estariam sendo intermediados pelas expectativas de ambos. A expectativa do cliente e a condição de acessibilidade ao serviço influencia fortemente o resultado psicossocial apresentado pela clientela, conforme sugere Tanaka (1990).

A ampla utilização da abordagem de Estrutura-Processo-Resultado no setor saúde tem mostrado maior factibilidade e uso nos seus componentes de Estrutura e Processo. A abordagem de Resultado tem sido menos explorada, havendo necessidade de se conhecerem meIhor os mecanismos e a importância da Estrutura e do Processo na obtenção do Resultado.

A utilização de métodos e de instrumentos quantitativos originários da área de administração tem permitido avanços nas práticas de avaliação de Estrutura e Processo dos serviços de saúde, o que tem resultado em decisões essencialmente gerenciais que objetivam a busca da eficiência e da otimização de recursos.

A avaliação do Resultado, na sua dimensão "fisiopatológica", isto é, nos efeitos concretos na história natural das doenças, é talvez a mais importante e menos atingível, quer pela diversidade e interação das variáveis que determinam o nível de saúde coletiva, quer pelo tempo necessário para mudanças neste nível de saúde, tomando-se por base processos de intervenção, principalmente no caso de doenças crônicas. Algumas doenças infecciosas - como as imunopreviníveis - são exceções, e a avaliação de seus programas de controle tem sido mais direcionada à análise de resultados do que de estrutura e de processo.

É na sua dimensão "psicossocial" que o resultado tem sido avaliado com maior freqüência, isto é, naquela que diz respeito aos efeitos ocorridos no nível da interação entre usuários e provedores.

Um programa de controle da hipertensão necessitará de anos para alterar o perfil de morbi-mortalidade da população. Porém, indicadores como cobertura, concentração e aderência poderão avaliar este processo de intervenção em um curto período de tempo. A eficácia das atividades desenvolvidas - pressuposto para a implantação do programa - é melhor avaliada em estudos desenhados com esta finalidade específica. A escolha das drogas mais eficazes e das condutas mais adequadas ao controle da hipertensão provém dos ensaios clíni- 
cos, e a incorporação destas drogas e condutas em programas de intervenção deverá possibilitar um aumento da sua efetividade, ainda que os resultados "fisiopatológicos" e de impacto epidemiológico sejam observáveis apenas em longos períodos de tempo.

Esta dimensão do processo e de parte do resultado só é plenamente abordada valendose de estudos com base populacional, pois as informações obtidas a partir da demanda são, na maioria das vezes, altamente seletivas, ocultando um dos aspectos mais importantes da avaliação de serviços de saúde nos países em desenvolvimento, e mesmo naqueles desenvolvidos: a iniqüidade no acesso aos serviços de saúde. Dentre os estudos com base populacional, os inquéritos domiciliares vêm sendo utilizados há algum tempo nos países industrializados, como fonte de informação importante para conhecimento da situação de saúde da população, e do acesso aos serviços de saúde, fornecendo subsídios ao planejamento e à avaliação destes. Apesar da ampla possibilidade de uso da epidemiologia na avaliação de serviços de saú de, poucas são as publicações que relatam a aplicação prática deste enfoque - a maioria dos trabalhos apresentados se refere a estudos do tipo diagnóstico que foram utilizados essencialmente para identificação de necessidades ou relato de situação, e pouco para avaliação propriamente dita de ações de saúde (Campos, 1988).

No entanto, inquéritos domiciliares em nível local podem ser úteis para aprimorar a avaliação de Resultado, pois permitirão a construção de "linhas de base" (Campos, 1993). Estas servirão de parâmetros para a comparação tanto das condições de saú de, como das características de utilização dos serviços de saúde, resultando na emissão do juízo de valor, tão necessário à consecução dos objetivos da avaliação de serviços de saúde.

Um estudo de caso na região sudoeste da área metropolitana de São Paulo

\section{Introdução}

O presente estudo refere-se a dados levantados em um inquérito domiciliar realizado entre juIho de 1989 e junho de 1990 na região sudoeste da Grande São Paulo (Cesar et al., 1992), área

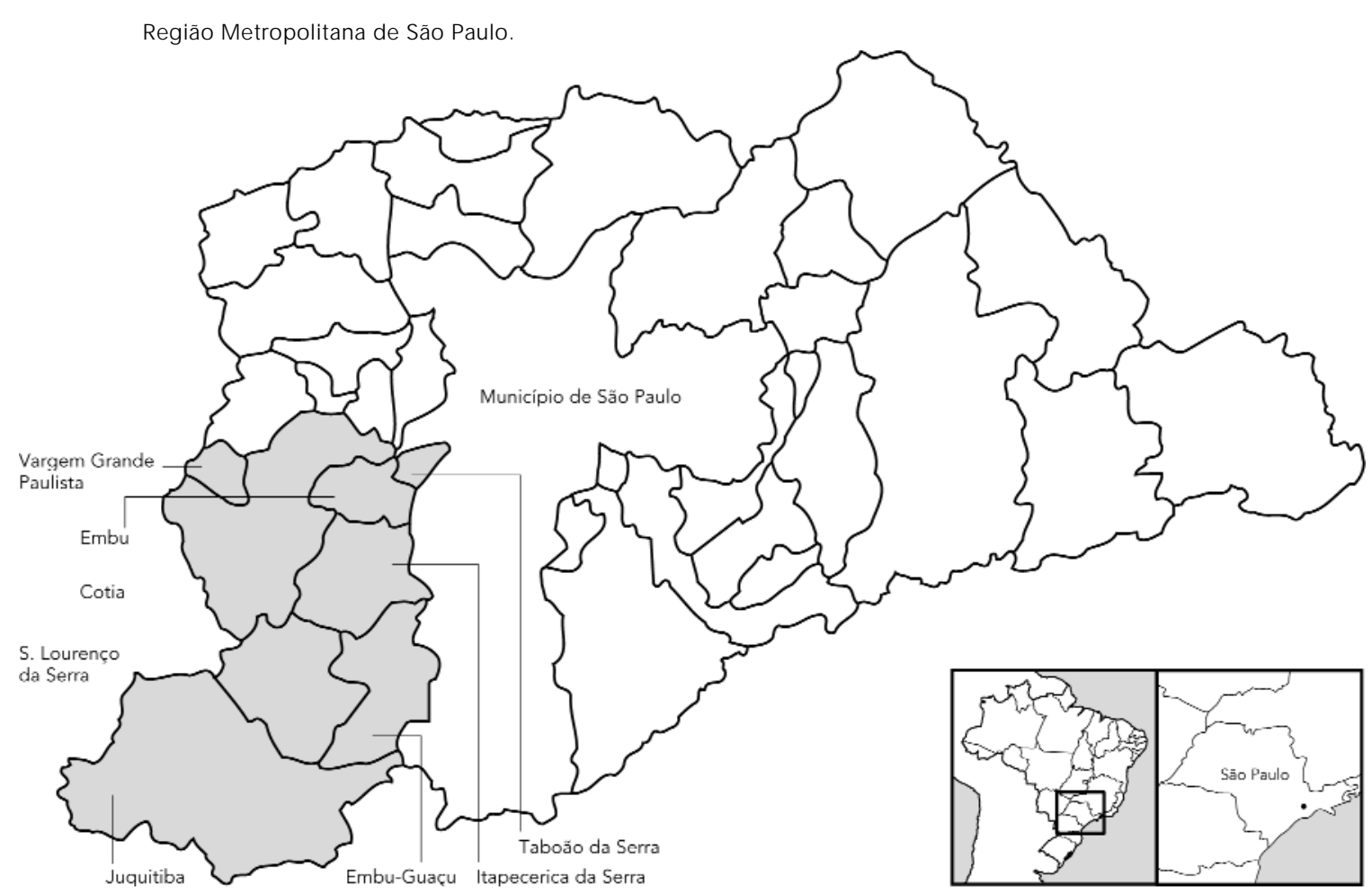


formada atualmente por oito Municípios - Taboão da Serra, Embu, Itapecerica da Serra, São Lourenço da Serra, Embu-Guaçu, Juquitiba, Cotia e Vargem Grande Paulista - com 552.672 habitantes, segundo o censo de 1991 (Figura 1). Durante o inquérito as pessoas foram entrevistadas sobre problemas de saúde, uso de serviços de saúde e condições de vida. Esta pesquisa foi desenvolvida tendo como objetivo principal subsidiar o planejamento e a avaliação dos serviços de saúde na área estudada, bem como permitir o desenvolvimento deste tipo de estudo em nosso meio, sob a perspectiva da administração dos serviços de saúde. Esta finalidade operacional orientou todo o desenvolvimento da pesquisa, de tal forma que fossem abordadas as questões consi deradas prioritárias ao processo de gerência dos serviços de saúde, que foi o interesse fundamental da Secretaria de Estado da Saúde com relação ao projeto, pois naquele momento ela vinha desenvolvendo um "Programa Metropolitano de Saúde" (Secretaria de Estado da Saúde, 1984), com implantação de "Módulos de Saúde" concepção semelhante aos Distritos de Saúde - como áreas programáticas de saúde.

As informações coletadas na pesquisa deveriam funcionar como uma "linha de base" para a implantação dos módulos de saúde, sendo que estudos posteriores permitiriam avaliar o processo de reorganização destes serviços. Na verdade, a pesquisa funcionou também como uma avaliação dos serviços existentes, na medida em que identificou os problemas de saúde da população estudada e as formas como foram solucionados ou orientados.

\section{Material e métodos}

A pesquisa trabalhou com uma amostra representativa da população residente em cada um dos municípios que compõem a região estudada, utilizando a técnica de "amostragem de área", com várias etapas (setor censitário, quarteirão, domicílio). Durante um período de 12 meses foram entrevistados 10.199 indivíduos.

O planejamento da amostra foi feito de maneira a permitir não só a análise individual de cada município, como também a dos diferentes grupos etários, inclusive daquel es numericamente pouco representados na população, como os menores de um e os maiores de cinqüenta anos (Holzer et al., 1985). Com estas características, o banco de dados formado possibilitou conhecer a realidade de cada município, além da área como um todo, assim como os problemas de saúde e as características de acesso aos serviços dos diferentes subgrupos populacionais, definidos por idade e sexo. Estes subgrupos populacionais (domínios amostrais) foram: menores de um ano de idade, um a seis anos, sete a 19 anos, 20 a 49 anos - sexo masculino; 20 a 49 anos - sexo feminino; 50 anos ou mais - sexo masculino e 50 anos ou mais - sexo feminino.

As características dos procedimentos amostrais utilizados exigem que seja feita uma ponderação dos dados antes da sua análise. No presente estudo, esta ponderação foi feita levando em conta os dados do censo do IBGE para 1991, e as tabelas anal isadas apresentam os números absolutos na amostra não ponderada e a distribuição percentual dos valores na amostra ponderada.

$\mathrm{O}$ instrumento de coleta de dados foi um questionário organizado em 14 blocos, sendo que cada um destes estudou questões correlatas. Os temas estudados foram: morbidade referida para os 15 dias anteriores à entrevista; referência de doença crônica ou de deficiência física; teste CAGE - indicativo de alcoolismo; consumo de medicamentos nos três dias anteriores à entrevista; utilização de serviços de saúde e de seguro-saúde; uso de serviços odontológicos; uso de serviços materno-infantis (pré-natal, parto e puericultura); imunizações; conhecimento sobre saúde-doença e opinião sobre os serviços utilizados; condição sócio-econômica dos indivíduos entrevistados maiores de sete anos; condição sócio-econômica dos chefes de família; caracterização da habitação.

Os questionários foram aplicados por entrevistadores com segundo grau completo, sem experiência prévia em saúde, que receberam treinamento inicial para a realização das entrevistas, sendo acompanhados, avaliados e reciclados durante todo o período da pesquisa.

Uma descrição detalhada da metodologia da pesquisa foi objeto de uma publicação específica (Cesar et al., 1996).

\section{Resultados}

As informações disponíveis no banco de dados permitem inúmeras análises, desde questões mais abrangentes, como o perfil de morbidade e a prevalência de doenças crônicas, até questões mais singulares, como razões para nãouso de serviços odontológicos, ou perfil social das crianças não vacinadas.

É importante ressaltar que essa área corresponde a uma área programática de saúde SUDS- 12 - e a análise dos dados aqui apresen- 
tada foi feita em função da avaliação da resolutividade dos serviços em nível municipal e da área programática.

Cerca de $30 \%$ das pessoas entrevistadas referiram algum problema de saúde nos 15 dias anteriores à entrevista, variando esta porcentagem nos diferentes grupos estudados. Os menores de um ano de idade e as mulheres com 50 anos ou mais foram os grupos que apresentaram as maiores proporções: 41,3\% e 48,7\% respectivamente; ao passo que o grupo de sete a 19 anos apresentou a menor proporção: $22,5 \%$. Dentre os indivíduos que apresentaram problema, a procura de ajuda decresceu com a idade, sendo que $75,61 \%$ dos menores de um ano que referiram algum problema procuraram algum tipo de ajuda; para as idades mais avançadas, mesmo na presença de algum problema, a procura foi menor, mostrando a existência de maior preocupação com os problemas de saúde das crianças de baixa idade. Este padrão de prevalência de problemas de saúde e procura de ajuda foi mais ou menos semeIhante para os diversos municípios estudados.

As porcentagens de morbidade referida foram semel hantes quando analisadas segundo a escolaridade dos chefes de família, porém a procura de ajuda aumentou com a escolaridade, sugerindo que esta procura poderia estar relacionada à maior possibilidade de acesso aos serviços.

A procura de ajuda foi em grande parte dirigida ao médico $(74,9 \%)$ e em menor proporção ao farmacêutico (9,9\%). A procura de outros profissionais de saúde ou de formas tradicionais, como benzedor, foi de apenas 2,6\%.
Uma proporção de $12,6 \%$ de procura de ajuda referiu-se a parentes, amigos ou vizinhos. Esta procura de ajuda apresentou variações com relação ao sexo e à idade, conforme pode ser visto na Tabela 1.

O tipo de serviço de saúde mais procurado para a morbidade de 15 dias foi Hospital ou Pronto Socorro (40,3\%), seguido por Unidade Básica de Saúde $(37,1 \%)$ e Clínicas (clínicas, consultórios particulares, ambulatórios, serviços de convênio de saúde) (20,1\%). Houve uma diferença acentuada destes percentuais para o grupo masculino de 20 a 49 anos de idade, que fez o menor uso de UBS $(13,1 \%)$ e o maior de Hospital ou Pronto Socorro (55,6\%).

A maioria dos entrevistados $(90,5 \%)$ não pagou diretamente pelo serviço utilizado para a morbidade de 15 dias, e apenas uma proporção de 7,5\% pagou integralmente.

Dentre os serviços utilizados pelos entrevistados que não pagaram diretamente, $68 \%$ foram serviços públicos ou conveniados com o setor público; $24,1 \%$, convênio-empresa; $7,9 \%$, outras alternativas, como serviço médico de empresas, de sindicatos ou associações de categorias profissionais. Foi utilizado inclusive seguro médico privado, numa porcentagem inferior a 1\% (Tabela 2). Estas proporções variaram de acordo com o sexo e a idade, sendo que o setor público cobriu em maior proporção a população de 50 anos ou mais de idade, enquanto o convênio-empresa concentrou-se mais na população economicamente ativa e seus dependentes. A população masculina de 20 a 49 anos foi a que apresentou maior proporção de outras alternativas (15,3\%).

Tabela 1

Profissional procurado pelos entrevistados que apresentaram algum problema de saúde (morbidade de 15 dias), por sexo e faixa etária. SUDS-12, São Paulo, 1989-1990.

\begin{tabular}{|c|c|c|c|c|c|c|c|c|c|c|}
\hline \multirow[t]{3}{*}{ Sexo e faixa etária } & \multicolumn{8}{|c|}{ Profissionais } & \multicolumn{2}{|c|}{ Total } \\
\hline & \multicolumn{2}{|c|}{ Médico } & \multicolumn{2}{|c|}{ Farmacêutico } & \multicolumn{2}{|c|}{$\begin{array}{c}\text { Parente, amigo } \\
\text { ou vizinho }\end{array}$} & \multicolumn{2}{|c|}{ O utros * } & \multirow[b]{2}{*}{ (1) } & \multirow[b]{2}{*}{ (2) } \\
\hline & (1) & (2) & (1) & (2) & (1) & $(2)$ & (1) & $(2)$ & & \\
\hline$M / F<$ lano & $(236)$ & 89,0 & (7) & 2,4 & $(22)$ & 7,6 & (4) & 1,0 & (269) & 100,0 \\
\hline$M / F 1$ a 6 & (205) & 82,8 & (20) & 8,7 & (15) & 6,8 & (8) & 1,7 & $(248)$ & 100,0 \\
\hline M/F 7 a 19 & (150) & 69,9 & (19) & 8,6 & (37) & 18,6 & (7) & 2,9 & (213) & 100,0 \\
\hline M 20 a 49 & (133) & 69,6 & (34) & 13,3 & $(21)$ & 12,5 & (12) & 4,6 & $(200)$ & 100,0 \\
\hline F 20 a 49 & $(259)$ & 70,3 & (37) & 12,9 & $(46)$ & 14,0 & (9) & 2,8 & (351) & 100,0 \\
\hline M 50 ou mais & (205) & 85,8 & (14) & 5,3 & $(21)$ & 7,9 & (4) & 1,0 & (244) & 100,0 \\
\hline F 50 ou mais & $(284)$ & 83,9 & $(22)$ & 5,8 & $(29)$ & 9,2 & (7) & 1,1 & $(342)$ & 100,0 \\
\hline Total & $(1472)$ & 74,9 & (153) & 9,9 & (191) & 12,6 & (51) & 2,6 & (1867) & 100,0 \\
\hline
\end{tabular}

(1) Números na amostra não ponderada.

(2) Percentuais na amostra ponderada.

* Como "outros" estão agrupados dentistas, psicólogos, enfermeiros e demais profissionais da área da saúde,

bem como "benzedor ou curandeiro". 
Fonte pagadora da assistência à morbidade de 15 dias dos que não pagaram integralmente, por sexo e faixa etária, SUDS-12, São Paulo, 1989-1990.

\begin{tabular}{|c|c|c|c|c|c|c|c|c|}
\hline \multirow[t]{3}{*}{ Sexo e faixa etária } & \multicolumn{6}{|c|}{ Fonte Pagadora } & \multicolumn{2}{|c|}{ Total } \\
\hline & \multicolumn{2}{|c|}{ Convênio-empresa } & \multicolumn{2}{|c|}{ Serviço público } & \multicolumn{2}{|c|}{ O utras * } & \multirow[b]{2}{*}{ (1) } & \multirow[b]{2}{*}{$(2)$} \\
\hline & (1) & $(2)$ & $(1)$ & (2) & (1) & $(2)$ & & \\
\hline $\mathrm{M} / \mathrm{F}<1$ ano & $(42)$ & 21,9 & (168) & 73,3 & (6) & 4,8 & $(216)$ & 100,0 \\
\hline $\mathrm{M} / \mathrm{F} 1$ a 6 & (30) & 22,3 & (153) & 74,3 & (6) & 3,4 & (189) & 100,0 \\
\hline M/F 7 a 19 & $(21)$ & 19,0 & (113) & 73,7 & (9) & 7,3 & (143) & 100,0 \\
\hline M 20 a 49 & (25) & 28,5 & (83) & 56,2 & (13) & 15,3 & (121) & 100,0 \\
\hline F 20 a 49 & (53) & 33,5 & (161) & 57,9 & (11) & 8,6 & $(225)$ & 100,0 \\
\hline M 50 ou mais & (18) & 14,0 & $(150)$ & 77,9 & (13) & 8,1 & (181) & 100,0 \\
\hline F 50 ou mais & $(30)$ & 12,6 & (192) & 79,8 & (15) & 7,6 & $(237)$ & 100,0 \\
\hline Total & $(219)$ & 24,1 & $(1020)$ & 68,0 & (73) & 7,9 & (1312) & 100,0 \\
\hline
\end{tabular}

(1) Números absolutos na amostra não ponderada.

(2) Percentuais na amostra ponderada.

* Como "outras" estão agrupados serviços médicos de "empresas", "sindicatos e associaçãoes de categorias profissionais" e "seguro-saúde privado".

Tabela 3

Fonte pagadora da assistência à morbidade de 15 dias dos que não pagaram integralmente, por escolaridade do chefe da família, SUDS-12, São Paulo, 1989-1990.

\begin{tabular}{|c|c|c|c|c|c|c|c|c|}
\hline \multirow{3}{*}{$\begin{array}{l}\text { Escolaridade do chefe } \\
\text { da família }\end{array}$} & \multicolumn{6}{|c|}{ Fonte Pagadora } & \multicolumn{2}{|c|}{ Total } \\
\hline & \multicolumn{2}{|c|}{ Convênio-empresa } & \multicolumn{2}{|c|}{ Serviço público } & \multicolumn{2}{|c|}{ O utras * } & \multirow[b]{2}{*}{ (1) } & \multirow[b]{2}{*}{$(2)$} \\
\hline & (1) & (2) & (1) & $(2)$ & $(1)$ & $(2)$ & & \\
\hline Nenhuma & $(25)$ & 19,9 & (199) & 75,6 & $(10)$ & 4,5 & $(234)$ & 100,0 \\
\hline Até 4 anos & (83) & 18,4 & $(531)$ & 74,8 & (31) & 6,8 & $(645)$ & 100,0 \\
\hline De 5 a 8 anos & $(48)$ & 24,7 & $(221)$ & 69,4 & (9) & 5,9 & $(278)$ & 100,0 \\
\hline De 9 a 11 anos & $(36)$ & 49,2 & (33) & 29,1 & $(14)$ & 21,7 & (83) & 100,0 \\
\hline Doze anos ou mais & $(13)$ & 60,8 & (3) & 7,1 & (7) & 32,1 & $(23)$ & 100,0 \\
\hline Total & $(205)$ & 23,6 & (987) & 68,4 & $(71)$ & 8,0 & (1263) & 100,0 \\
\hline
\end{tabular}

(1) Números absolutos na amostra não ponderada.

(2) Percentual na amostra ponderada.

* Como "outras" estão agrupados serviços médicos de "empresas", "sindicatos", "sindicatos e associações de categorias profissionais" e "seguro-saúde privado".

Esta forma de pagamento dos serviços utilizados para a morbidade de 15 dias variou também com a escolaridade do chefe de família, e nos diferentes municípios.

Com relação à escolaridade, há um nítido decréscimo da participação do setor público com o aumento da escolaridade, ocorrendo o inverso com o convênio-empresa e com as outras alternativas (Tabela 3).

A participação do setor público, convênioempresa e outras alternativas variou também entre os diversos municípios, com maior participação do segundo em Cotia (31\%) e Taboão da Serra (26,1\%). Já o setor público teve grande participação no Município de Juquitiba (95,5\%).
Uma alta proporção de gestantes (88,9\%) não pagou diretamente o serviço utilizado para pré-natal, ao passo que 8,5\% pagou integralmente por estes serviços. Estas proporções variaram com a escolaridade da mãe, conforme pode ser visto na Tabela 4. Dentre as que não pagaram, 70,51\% dos serviços utilizados foram públicos ou conveniados com o setor público; 25,0\%, convênio-empresa e 4,4\%, outras alternativas (Tabela 5). A distribuição destes percentuais também variou com o nível de escolaridade da mãe: com o aumento deste, diminuiu a participação do setor público e aumentou a do convênio-empresa e de outras alternativas, inclusive a do seguro-saúde particular. 
Pagamento da consulta de pré-natal e parto, por escolaridade da mãe, SUDS-12, São Paulo, 1989-1990.

\begin{tabular}{|c|c|c|c|c|c|c|c|c|}
\hline & & & \multicolumn{5}{|c|}{ Escolaridade da Mãe } & \multirow[t]{2}{*}{ Total } \\
\hline & & & Nenhuma & Até 4 anos & $\begin{array}{l}\text { De } 5 \text { a } \\
8 \text { anos }\end{array}$ & $\begin{array}{c}\text { De } 9 \text { a } \\
11 \text { anos }\end{array}$ & $\begin{array}{l}12 \text { anos } \\
\text { ou mais }\end{array}$ & \\
\hline \multirow{8}{*}{$\begin{array}{l}\text { Pagamento da } \\
\text { consulta de pré-natal }\end{array}$} & Não & $(1)$ & (37) & $(266)$ & $(243)$ & (84) & $(14)$ & $(644)$ \\
\hline & & $(2)$ & 100,0 & 93,3 & 88,1 & 81,1 & 66,5 & 88,9 \\
\hline & Sim, & $(1)$ & - & $(7)$ & (9) & $(4)$ & - & $(20)$ \\
\hline & Parcialmente & $(2)$ & - & 2,6 & 3,4 & 2,2 & - & 2,6 \\
\hline & Sim, & $(1)$ & - & $(11)$ & $(23)$ & (19) & (8) & $(61)$ \\
\hline & Integralmente & $(2)$ & - & 4,1 & 8,5 & 16,7 & 33,5 & 8,5 \\
\hline & Total & (1) & (37) & $(284)$ & $(275)$ & $(107)$ & $(22)$ & $(725)$ \\
\hline & & $(2)$ & 100,0 & 100,0 & 100,0 & 100,0 & 100,0 & 100,0 \\
\hline \multirow[t]{8}{*}{ Pagamento do parto } & Não & $(1)$ & $(37)$ & $(241)$ & $(240)$ & (82) & (13) & (613) \\
\hline & & $(2)$ & 92,2 & 85,0 & 87,7 & 77,4 & 65,0 & 84,6 \\
\hline & Sim & (1) & (1) & $(21)$ & (10) & (10) & (4) & (46) \\
\hline & Parcialmente & $(2)$ & 5,7 & 7,7 & 4,6 & 6,3 & 17,5 & 6,5 \\
\hline & Sim, & (1) & (1) & (24) & (26) & (16) & (4) & (71) \\
\hline & Integralmente & $(2)$ & 2,1 & 7,3 & 7,7 & 16,3 & 17,5 & 8,9 \\
\hline & Total & (1) & (39) & $(286)$ & $(276)$ & $(108)$ & $(21)$ & $(730)$ \\
\hline & & $(2)$ & 100,0 & 100,0 & 100,0 & 100,0 & 100,0 & 100,0 \\
\hline
\end{tabular}

(1) Números absolutos na amostra não ponderada.

(2) Percentuais na amostra ponderada.

A utilização dos serviços de saúde para o parto é muito semelhante, mostrando uma grande parcela que utilizou serviços públicos, seguidos pelo convênio-empresa, bem como um diferencial grande por escolaridade da mãe com relação aos serviços utilizados (Tabelas 4 e 5).

Estes dados mostram o quanto a variável escolaridade da mãe esteve associada ao acesso a outros serviços de saúde que não os públicos.

Uma questão analisada, de importância para a política de saúde na área, foi a localização dos serviços utilizados, e em particular a dos serviços públicos.

Com relação à morbidade referida de 15 dias (Figura 2), Itapecerica apresentou a menor proporção da população atendida pelos serviços públicos no próprio Município (54,69\%), ea maior proporção atendida no Município de São Paulo (34,45\%). Juquitiba e Embu-Guaçu apresentaram as maiores proporções de atendimento no próprio Município (95,13\% e 95,26\%) e as menores proporções de atendimento no Município de São Paulo (2,74\% e 4,4\%).

Os Municípios de Embu e Itapecerica foram os únicos nos quais parte da população foi atendida em Municípios vizinhos pertencentes à mesma área programática - SUDS-12 -, sendo estas proporções de 9,40\% para Itapecerica da Serra, e 18,95\% para o Embu.
Para o pré-natal (Figura 3), o Município de Embu apresentou a menor proporção de serviços utilizados no próprio Município (39,1\%), e Juquitiba, a maior proporção (91,1\%). Para a área como um todo, cerca de $27 \%$ das consultas de pré-natal foram feitas no Município de São Paulo, com a proporção maior $(42,1 \%)$ correspondendo a gestantes residentes em Itapecerica da Serra, e a menor $(2,2 \%)$ a gestantes residentes em Juquitiba.

Grande parte dos partos das usuárias dos serviços públicos $(61,8 \%)$ foram realizados no Município de São Paulo. Para o Município de Embu-Guaçu esta proporção foi de $91,9 \%$ e para Juquitiba, ainda que distante $70 \mathrm{Km}$ de São Paulo, a proporção foi de 39,5\%. A área de Cotia eVargem Grande Paulista, na região estudada, única com infra-estrutura hospitalar própria, apresentou uma porcentagem de 19,1\% (Figura 4).

\section{Discussão}

Estas informações descritas indicam algumas características importantes dos serviços de saúde da área estudada:

a) A população, em sua maioria, procurou ajuda para os problemas de saúde referidos 
Fonte pagadora da consulta de pré-natal e parto dos que não pagaram integralmente, por escolaridade da mãe. SUDS-12, São Paulo, 1989-1990.

\begin{tabular}{|c|c|c|c|c|c|c|c|c|}
\hline & & & \multicolumn{5}{|c|}{ Escolaridade da Mãe } & \multirow[t]{2}{*}{ Total } \\
\hline & & & Nenhuma & $\begin{array}{l}\text { Até } 4 \\
\text { anos }\end{array}$ & $\begin{array}{l}\text { De } 5 \text { a } \\
\text { anos }\end{array}$ & $\begin{array}{l}\text { De } 9 \text { a } \\
11 \text { anos }\end{array}$ & $\begin{array}{l}12 \text { anos } \\
\text { ou mais }\end{array}$ & \\
\hline \multirow{8}{*}{$\begin{array}{l}\text { Fonte pagadora da } \\
\text { consulta de pré-natal }\end{array}$} & Convênio & (1) & (6) & $(54)$ & (65) & (33) & (8) & (166) \\
\hline & empresa & (2) & 10,6 & 21,6 & 24,7 & 35,4 & 60,0 & 25,1 \\
\hline & Serviço & (1) & $(31)$ & $(215)$ & (178) & $(45)$ & - & $(469)$ \\
\hline & público & (2) & 89,4 & 77,0 & 71,3 & 54,9 & 0 & 70,5 \\
\hline & O utras * & (1) & - & (4) & (7) & (10) & $(6)$ & $(27)$ \\
\hline & & $(2)$ & - & 1,4 & 4,0 & 9,7 & 40,0 & 4,4 \\
\hline & Total & (1) & (37) & $(273)$ & $(250)$ & (88) & (14) & $(662)$ \\
\hline & & (2) & 100,0 & 100,0 & 100,0 & 100,0 & 100,0 & 100,0 \\
\hline \multirow{8}{*}{$\begin{array}{l}\text { Fonte pagadora } \\
\text { do parto }\end{array}$} & Convênio & (1) & $(5)$ & (49) & $(61)$ & $(27)$ & (8) & $(150)$ \\
\hline & empresa & (2) & 9,3 & 19,9 & 23,1 & 28,3 & 44,9 & 22,4 \\
\hline & Serviço & (1) & (33) & $(209)$ & $(180)$ & (55) & - & $(477)$ \\
\hline & público & (2) & 90,7 & 79,0 & 73,2 & 63,2 & 0 & 73,0 \\
\hline & O utras * & (1) & - & (3) & (7) & (10) & (9) & (29) \\
\hline & & (2) & - & 1,1 & 3,7 & 8,5 & 55,1 & 4,6 \\
\hline & Total & (1) & (38) & $(261)$ & $(248)$ & (92) & (17) & (656) \\
\hline & & (2) & 100,0 & 100,0 & 100,0 & 100,0 & 100,0 & 100,0 \\
\hline
\end{tabular}

(1) Números absolutos na amostra não ponderada.

(2) Percentuais na amostra ponderada.

* "O utras" correspondeu principalmente a "seguro médico privado" e uma pequena proporção distribuiu-se em diversas outras alternativas.

nos 15 dias anteriores à entrevista, em serviços institucionais - hospitais, prontos-socorros, Unidades Básicas de Saúde e consultórios ou clínicas - e em menor proporção procurou farmácias. O profissional mais procurado foi, na grande maioria das vezes, o médico, e em segundo lugar o farmacêutico. Houve pouca procura por outros profissionais de saúde, como psicólogos ou dentistas. Foi inexpressiva a referência à procura de profissionais ligados a formas tradicionais de tratamento como benzedor, parteira e outros.

b) Tanto para a morbidade de 15 dias, como para a atenção pré-natal e principalmente o parto, a população em sua maioria utilizou-se de serviços públicos de saúde, mostrando assim a importância deste setor como provedor da assistência médica nesta área. A principal alternativa ao setor público foi o convênioempresa, que cobriu uma parcela expressiva da população. As demais alternativas, incluindo o seguro-saúde privado, representaram uma parcela muito pequena dos serviços utilizados.

c) Os dois maiores provedores da atenção médica na área - o setor público e o convênio-empresa - foram utilizados por populações diferentes, do ponto de vista sócio-econômico e demográfico. O setor público cobriu propor- cionalmente mais a população idosa, e o convênio-empresa, a população economicamente ativa e seus dependentes. Do ponto de vista sócio-econômico, abordado aqui através da escolaridade do chefe da família ou da escolaridade da mãe, os diferenciais são ainda maiores, sendo a clientela do convênio-empresa representada basicamente pela população de escolaridade mais alta, traduzindo possivelmente mão-de-obra mais qualificada vinculada a empresas de maior porte e com convênios de saúde para seus funcionários. A população de baixa escolaridade foi assistida, em sua maioria, pelo setor público.

d) Esta população da área estudada, que em sua maioria é dependente dos serviços públicos de saúde, foi em grande parte atendida fora de seu município de residência, tanto para a morbidade de 15 dias como para o pré-natal. Grande parcela dos partos foi realizada no Município de São Paulo, mesmo para as usuárias dos serviços públicos.

O Embu foi praticamente o único Município a utilizar serviços de outros do SUDS- 12 .

Estes dados indicam uma baixa capacidade do setor público local de atender a população dependente dele, uma grande necessidade de ampliação dos serviços materno-infantis e apontam para as dificuldades de execução de 
Localização dos serviços públicos de saúde ${ }^{1}$ utilizados para consulta (morbidade de 15 dias), por município do SUDS-12, São Paulo, 1989-1990.

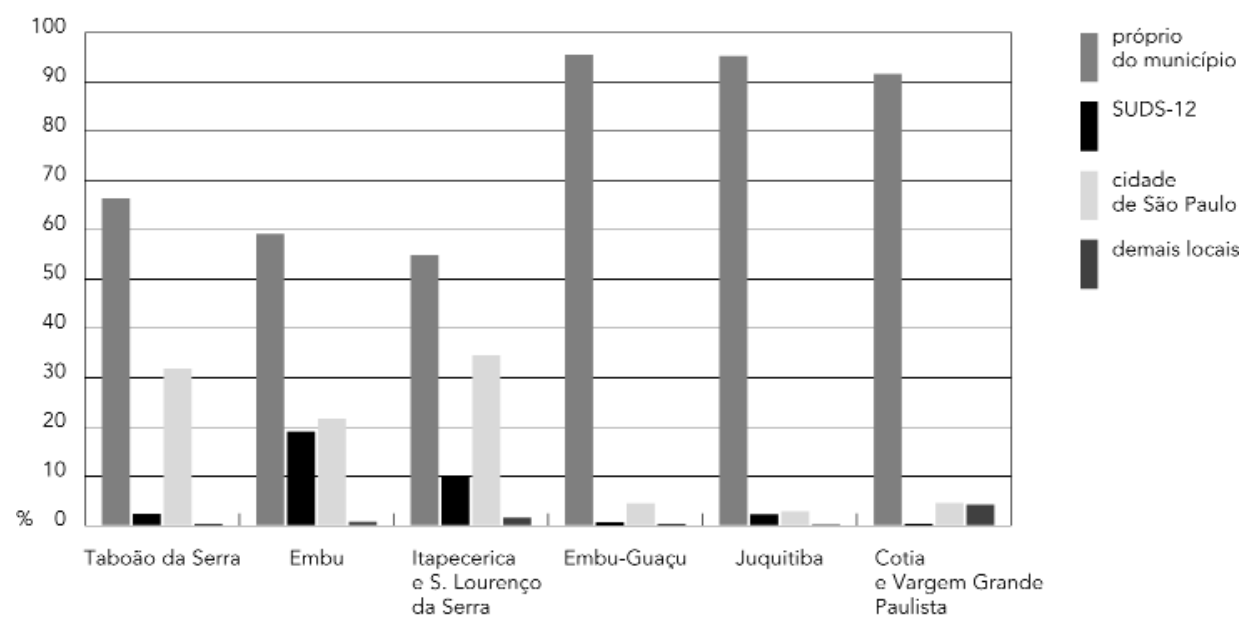

(1) Serviços próprios ou conveniados.

Figura 3

Localização dos serviços públicos de saúde ${ }^{1}$ utilizados para consulta de pré-natal, por município do SUDS-12, São Paulo, 1989-1990.

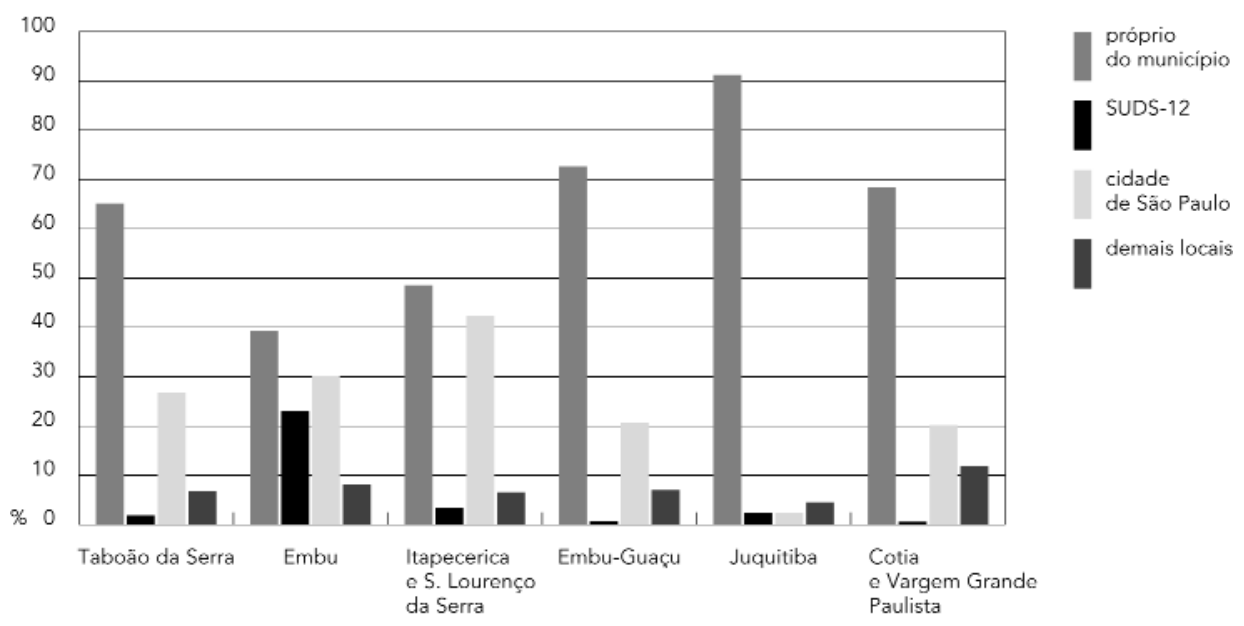

(1) Serviços próprios ou conveniados.

programas locais de saúde, em face das baixas coberturas existentes e da grande evasão para a procura de serviços de saúde em outros municípios, principalmente no de São Paulo.

e) O padrão de uso de serviços da área estudada reflete as características e tendências da atual situação do sistema de saúde no país. O crescimento do setor privado, principalmente da Medicina de Grupo, vem ocorrendo em grande parte através de convênios com empresas. Este setor privado cobre primordialmente a população economicamente ativa e seus dependentes, bem como destina-se a parcelas de melhor situação sócio-econômica. Este fato reforça a situação de dependência - da população idosa e das famílias de baixa renda - em relação ao setor público de saúde. É importante ressaltar que, nesta área estudada, mais de $50 \%$ 


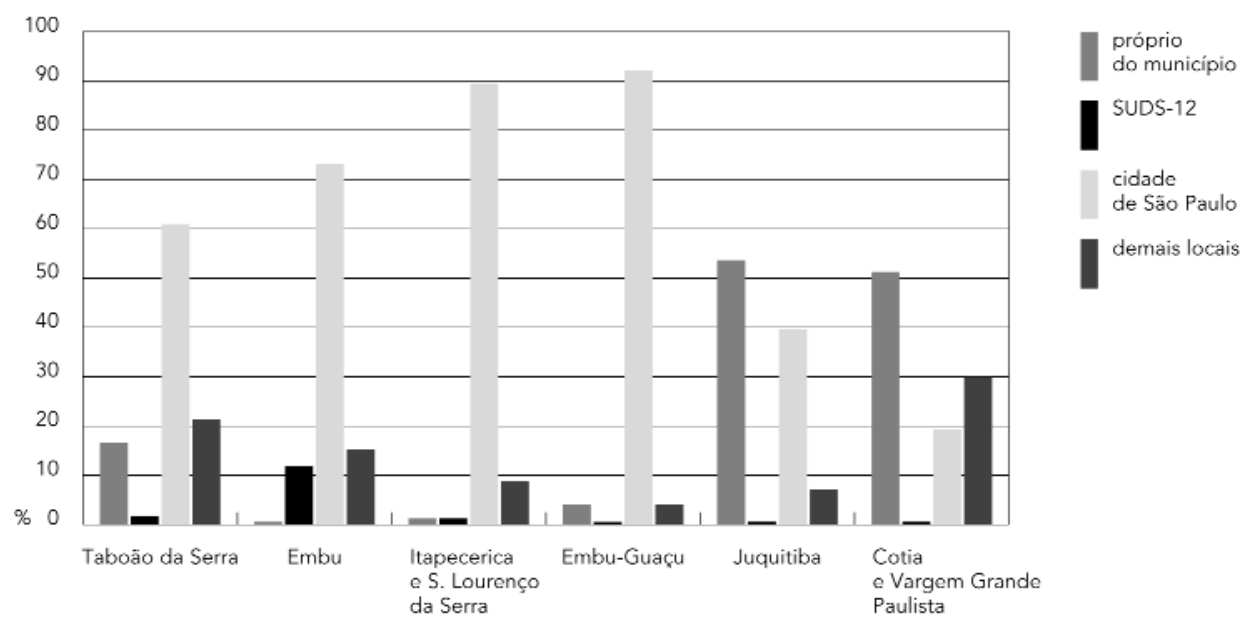

(1) Serviços próprios ou conveniados.

da população são de baixa escolaridade (até quatro anos) eque, a exemplo do que vem ocorrendo no país como um todo, há um crescimento da população idosa.

Embora a descentralização e municipalização dos serviços de saúde venha sendo apresentada como diretriz há vários anos, os dados mostram ainda a grande centralização existente na Grande São Paulo, mesmo para cuidados de prénatal e parto hospitalar, deixando grandes áreas desassistidas - como o SUDS-12 -, com cerca de 600.000 habitantes. A necessidade de deslocamentos à procura de serviços de pré-natal e parto pode causar uma repressão de demanda, com menor número de consultas de pré-natal, dificuldade de internação no momento do parto e, conseqüentemente, aumento do risco de morbi-mortalidade materna e perinatal.

\section{Considerações Finais}

Os inquéritos de saúde permitem avaliar com clareza o padrão de utilização dos serviços de saúde, identificando, também, as dificuldades de acesso aos mesmos.

A análise das informações geradas através destes inquéritos não só permite uma “linha de base" para planejamento de saúde e avaliação posterior, como também é um elemento importante de avaliação dos serviços existentes, na medida em que descreve elementos essenciais a esta análise.
A caracterização sócio-econômica e demográfica da população permite, ainda, caracterizar a forma diferenciada pela qual os distintos grupos populacionais utilizam os serviços de saúde.

Esses estudos com base populacional permitem definir com maior especificidade as diretrizes e as estratégias necessárias para a implantação de um sistema de saúde, na busca de maior eqüidade no atendimento às necessidades de saúde da população.

Devido ao custo operacional e à complexidade metodológica dos inquéritos domiciliares, há necessidade de uma avaliação prévia sobre o modelo mais adequado às diferentes situações específicas.

As informações necessárias em nível de Secretarias de Estado, grandes municípios ou de áreas programáticas - como Distritos de Saúde são diferentes daquelas necessárias em nível local, no que se refere tanto ao conteúdo das questões estudadas, quanto à precisão metodológica. A periodicidade dos levantamentos também deve ser variável conforme o tipo de estudo.

Estudos mais abrangentes, e com maior precisão metodológica, devem fornecer informações sobre padrões gerais de morbidade e de acesso aos serviços de saúde, sendo de maior utilidade para as decisões políticas. Estudos menos abrangentes e mais viáveis de serem executados em nível local devem responder às questões mais específicas, necessárias à gerência local dos serviços de saúde. 


\section{Referências}

CALSING, E., 1982. Diretrizes para uma sistemática de avaliação. Elementos para uma discussão. III Curso de Política e Planejamento Local. Brasília. (mimeo.)

CAMPOS, C. E. A., 1993. Os inquéritos de saúde sob a perspectiva do planejamento. Cadernos de Saúde Pública, 9:190-200.

CAMPOS, F. E., 1988. Resolutividade: Uma Aproximação da Avaliação Qualitativa de Serviços deSaúde. Tese de Doutorado, Rio de Janeiro: Departamento de Planejamento, Fundação Oswaldo Cruz.

CESAR, C .L. G., FIGUEIREDO, G. M.; WESTPHAL, M. F.; CARDOSO, M. R. A.; COSTA, M. Z. A. \& GATTÁS, V. L., 1992. Morbidade referida e utilização de serviços de saúde no ERSA de Itapecerica da Serra, São Paulo. Relatório da Pesquisa. São Paulo: Faculdade de Saúde Pública, USP/Secretaria de Estado da Saúde de São Paulo.

CESAR, C. L. G.; FIGUEIREDO, M. G.; WESTPHAL, M. F.; CARDOSO, M. R. A.; COSTA, M. Z. A. \& GATTÁS, V. L. 1996. Morbidade referida e utilização de serviços de saúde em municípios da Grande São Paulo, 1989-1990 - metodologia. Revista de Saúde Pública, 30(2):153-60.
CONSTANZO, G. A. \& VERTINSKY, I., 1975. Measuring the quality of health care: a decision oriented tipology. Medical Care, 13:417-431.

DONABEDIAN A., 1980. Explorations in Quality Assessment and Monitoring: The Definition of Quality and Approaches to its Assessment. Ann Harbor: Michigan $\mathrm{H}$. A. Press.

FRENK, J. O. \& MOHR, P. M., 1985. Evaluación de Tecnología y Calidad dela Atención en Salud. Washington: OPS.

HOLZER, C. E. et al., 1985. Sampling the house hold population. In: Epidemiological Field Methods in Psychiatry (W. W. Eaton \& L. G. Kessler, ed.), pp. 23-48, Orlando: Academic Press.

OMS/OPS (Organización Mundial de la Salud/Organización Panamericana de Salud), 1989. Evaluación de Servicios de la Salud Materno-Infantil. Castaballeda, Venezuela, 24-28 de agosto de 1987. Washington D. C.: OMS/OPS.

PABON, H., 1985. Evaluación de Servicios deSalud. 2a ed., Cali: XYZ.

SECRETARIA DE ESTADO DA SAÚDE, 1984. Programa Metropolitano de Saúde. São Paulo. (mimeo.).

TANAKA, O., 1990. Análise da utilização pela clientela de uma unidade ambulatorial da Secretaria de Saúde do Município de São Paulo (SP-Brasil). Revista de SaúdePública, 24: 60-68. 